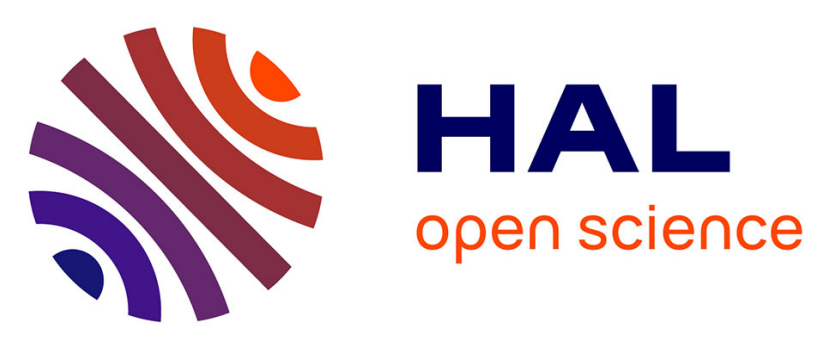

\title{
Synthesis of squaraine-based alternated $\pi$-conjugated copolymers: from conventional cross-coupling reactions to metal-free polycondensation
}

Jules Oriou, Feifei Ng, Georges Hadziioannou, Guillaume Garbay, Mélanie Bousquet, Eric Cloutet, Cyril Brochon

\section{To cite this version:}

Jules Oriou, Feifei Ng, Georges Hadziioannou, Guillaume Garbay, Mélanie Bousquet, et al.. Synthesis of squaraine-based alternated $\pi$-conjugated copolymers: from conventional cross-coupling reactions to metal-free polycondensation. Polymer Chemistry, 2014, 5 (24), pp.7100-7108. 10.1039/C4PY00896K . hal-02922842

\section{HAL Id: hal-02922842 \\ https://hal.science/hal-02922842}

Submitted on 28 Aug 2020

HAL is a multi-disciplinary open access archive for the deposit and dissemination of scientific research documents, whether they are published or not. The documents may come from teaching and research institutions in France or abroad, or from public or private research centers.
L'archive ouverte pluridisciplinaire HAL, est destinée au dépôt et à la diffusion de documents scientifiques de niveau recherche, publiés ou non, émanant des établissements d'enseignement et de recherche français ou étrangers, des laboratoires publics ou privés. 


\section{ARTICLE}

Cite this: DOI: $10.1039 /$ xoxxooooox

Received ooth January 2014 Accepted ooth January 2014

DOI: 10.1039/xoxxooooox

www.rsc.org/

\section{Synthesis of Squaraine-based alternated $\pi$-conjugated copolymers: from conventional cross-coupling reactions to metal-free polycondensation}

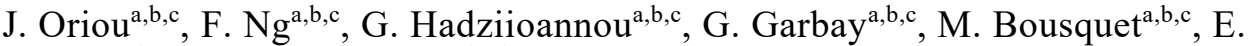 \\ Cloutet $^{\mathrm{a}, \mathrm{b}, \mathrm{c} \dagger}$ and C. Brochon ${ }^{\mathrm{a}, \mathrm{b}, \mathrm{c} \dagger}$,
}

Low band-gap $\pi$-conjugated copolymers based on squaraine units alternated with thiophene or benzothiadiazole moieties were synthesized through Suzuki and Stille cross-coupling reactions, and through a metal-free polycondensation process using squaric acid. The metal-free polymerizations afforded higher molecular weights polymers, showing bright future for the synthesis of small band-gap $\pi$-conjugated polymers via less cost-effective and more environmental-friendly procedures. Additionally, the synthesized materials showed encouraging optoelectronic properties.

\section{Introduction}

Due to their strong and red-shifted light absorption, organic dyes have been widely investigated as semiconducting materials in light-emission or light-harvesting devices. ${ }^{1-8}$ These dyes consist of $\pi$-conjugated small organic molecules, and usually present very planar structures which favor enhanced intermolecular interactions in the solid state. ${ }^{8,9}$ Additionally, they represent attractive building blocks for conjugated polymers employed in similar applications. The main advantage of polymers over small molecules lies in their easy processability when suitably functionalized, leading to the targeted low-cost organic electronic devices. ${ }^{10,11}$ Indeed small molecules may require unstraightforward technique to form homogeneous films such as vacuum deposition, ${ }^{12}$ which can be hard to transpose on large surfaces. Various dyes were used as monomers for the synthesis of $\pi$-conjugated polymers, such as diketopyrrolopyrrole, isoindigo or boron-dipyrromethene (BODIPY). ${ }^{6,13-15}$ Amongst them, squaraine (SQ) dyes attracted much attention due to their remarkable donor-acceptor-donor structure, leading to molecules with a small band-gap. ${ }^{16-21}$ Synthesis of $\pi$-conjugated polymers containing squaraine units is a promising strategy which was successfully employed to achieve materials with small band-gaps. ${ }^{22-28}$ The first example was reported by Treibs and co-workers, who described the preparation of an insoluble material obtained from pyrrole and squaric acid. ${ }^{16}$ However, no further investigation could be performed due to its unsoluble nature. Since then, variety of $\mathrm{N}$ alkyl or 3-alkyl substituted derivatives were developed, showing improved solubility and optical band-gap around 1.8
eV. ${ }^{29}$ Numerous research efforts were focused to further decrease the band-gap and it was found that extension of the conjugation and inclusion of electron-rich units in the backbone accomplished the desired effect. ${ }^{30}$ By enhancing the planarity and electron-donating character of difunctional monomers, Havinga et al. achieved very small band-gap polysquaraines. ${ }^{31}$ If a squaraine-based monomer is appropriately functionalized, polysquaraines can be obtained via classical synthetic routes such as Yamamoto or Suzuki coupling reactions. For instance, Lambert and co-workers developed an indole-based squaraine dye bearing bromides that was subsequently polymerized by Yamamoto coupling. ${ }^{24}$ The resulting polymer showed an optical band-gap around $1.6 \mathrm{eV}$ in film. Other research groups synthesized similar materials alternating the squaraine moiety with other conjugated subunits through Suzuki crosscoupling. ${ }^{28,} 32$ Based on the reported data, it was concluded that increasing the electron-withdrawing strength of the unit inserted between the squaraine moieties induced an increase of the band-gap, also confirmed by the blue-shift in absorption between the phenylene $\left(\lambda_{\max }{ }^{\text {sol }}=681 \mathrm{~nm}\right)$ and tetrafluorophenylene $\left(\lambda_{\max }{ }^{\text {sol }}=614 \mathrm{~nm}\right)$ linkers. However this effect could be attributed to a stronger twist angle between units when replacing hydrogen by fluorine atoms. On the contrary, only a slight red-shift could be observed when using electrondonating units. Generally, light absorption of these materials is blue-shifted compared with the non-bridged polymer $\left(\lambda_{\max }{ }^{\text {sol }}=\right.$ $738 \mathrm{~nm}$ ). This was attributed to a strong torsion angle induced by the spacing units. 
It is noteworthy that $\pi$-conjugated polysquaraines can also be obtained via the condensation of an appropriate monomer with squaric acid, as demonstrated by the early work of Treibs et al. for instance, ${ }^{16}$ and later by Ajayaghosh and co-workers. ${ }^{33,} 34$ The great advantage of this approach is that no transition metal catalyst is required. Moreover, the condensation of squaric acid with electron-rich molecules is less sensitive than metal-based couplings, and can be driven by a removal of the produced water during the reaction.

In this context, we developed two copolymers alternating squaraine sub-units with either the electron-donating thiophene moiety or the electron-withdrawing benzothiadiazole motif in order to provide a better understanding on the behaviour of squaraine units included in a $\pi$-conjugated backbone. This work particularly aimed to compare two polymerization pathways leading to such polysquaraine derivatives by classical Stille or Suzuki cross-coupling reactions and metal-free polycondensation via the use of squaric acid.

\section{Experimental}

\section{Materials and methods}

Tetrahydrofuran (THF), methylene chloride $\left(\mathrm{CH}_{2} \mathrm{Cl}_{2}\right)$, toluene and dimethylsulfoxide (DMSO) were purified from a solvent purification system (MBraun MB-SPS-800) prior to use. Dioxane was dried over calcium hydride $\left(\mathrm{CaH}_{2}\right)$ and distilled prior to use. 2,1,3-Benzothiadiazole-4,7-bis(boronic acid pinacol ester) was purified by sublimation at $160^{\circ} \mathrm{C}$ under vacuum prior to use. Squaric acid was recrystallized in boiling water and then rinsed with cold water and acetone, followed by drying under vacuum at $40^{\circ} \mathrm{C}$ overnight prior to use. Unless otherwise specified, all other solvents and chemicals were purchased from commercial suppliers (Alfa Aesar, SigmaAldrich, TCI, Acros Organics, Scharlau, Orgalight or Strem Chemicals) and used as received.

\section{Characterizations}

The precursors and polymers were characterized by IR, HRMS, ${ }^{1} \mathrm{H}$ and ${ }^{13} \mathrm{C}$ NMR in order to determine their chemical structures. NMR spectra were recorded on a Bruker $400 \mathrm{MHz}$ spectrometer from a deuterated chloroform or dimethyl sulfoxide $\left(\mathrm{CDCl}_{3}\right.$ or DMSO-d6) solution.

IR spectra were recorded with Bruker Tensor 27 spectrometer using a $0.6 \mathrm{~mm}$-diameter beam and a $4 \mathrm{~cm}^{-1}$ resolution. Samples were analyzed with the attenuated total reflexion (ATR) method.

A Kofler bench (Wagner\&Munz Heizbank system Kofler type wme) was used to obtain melting points with $\mathrm{a} \pm 2{ }^{\circ} \mathrm{C}$ precision.

High resolution mass spectroscopy analyses were performed on an AutoSpec-Waters spectrometer (EI).

Molecular weights of the polymers were measured by SEC at $30^{\circ} \mathrm{C}$ with $\mathrm{CHCl}_{3}$ as eluent, using a Malvern Viscotek TDA max, and PS standards were used for calibration.
Absorption and photoluminescence spectra were recorded for a polymer solution $\left(0.01 \mathrm{~g} / \mathrm{L}\right.$ in $\left.\mathrm{CHCl}_{3}\right)$ and a polymer film casted from an $\mathrm{CHCl}_{3}$ solution $(10 \mathrm{~g} / \mathrm{L})$ using the spectrophotometers UV-3600, Shimadzu and Fluoromax-4, Horiba Scientific, respectively.

Energetic levels were evaluated by cyclic voltammetry in a glovebox in electrolyte solutions of $0.1 \mathrm{M}$ TBAPF6 in $\mathrm{CHCl}_{3}$. Platinum was used as both working and counter electrodes and a silver wire was used as reference electrode. The obtained potentials were recalibrated outside the glovebox using ferrocene and saturated calomel electrode (SCE) as reference.

For solar cell devices preparation, etched ITO glass substrates with a sheet resistance of $10 \Omega \mathrm{sq}^{-1}$ were used. After cleaning of ITO glass substrate in an ultrasonic bath with acetone, ethanol, iso-propanol for $15 \mathrm{~min}$. and treated in a $\mathrm{UV} / \mathrm{O}_{3}$ cleaner for 20 min., PEDOT:PSS was spin coated at a spin speed of $4000 \mathrm{rpm}$ for $40 \mathrm{~s}$ forming $50 \mathrm{~nm}$ thick layers. The samples were annealed for $1 \mathrm{~h}$ at $110{ }^{\circ} \mathrm{C}$. All procedures were performed in an inert-atmosphere glovebox of nitrogen $\left(\mathrm{O}_{2}\right.$ and $\left.\mathrm{H}_{2} \mathrm{O}<0.1 \mathrm{ppm}\right)$. Different blend ratio of PSQBT and PCBM were prepared by making $24 \mathrm{mg} / \mathrm{mL}$ solutions in $o$ dichlorobenzene (o-DCB). The photoactive layer (squaraine-based polymer:PCBM 1:1 wt \%) was spin-coated on the top of the PEDOT-PSS layer from $o$-dichlorobenzene (o-DCB) solutions at $50{ }^{\circ} \mathrm{C}$. The thickness of the photoactive layer was typically of $80 \mathrm{~nm}$. The Al cathode was thermally deposited $(100 \mathrm{~nm})$ through a shadow mask under high vacuum $\left(10^{-6} \mathrm{mbar}\right)$. The active areas of the devices were ca. $8.4 \mathrm{~mm}^{2}$. An annealing treatment was performed at $120^{\circ} \mathrm{C}$ after the cathode deposition during 20 minutes. The current density-voltage $(\mathrm{J}-\mathrm{V})$ characteristics were measured with a Keithley 4200 SCS under an illumination of $100 \mathrm{~mW} / \mathrm{cm}^{2}$ from a K.H.S. Solar Celltest 575 solar simulator with AM1.5 filters and in the dark.

\section{Syntheses of precursors and polymers}

\section{p-Bromophenylhydrazine hydrochloride (1)}

A mixture of p-bromoaniline $(34.51 \mathrm{~g}, 0.200 \mathrm{~mol}), 100 \mathrm{ml}$ of water and $50 \mathrm{ml}$ of hydrochloric acid $(36 \% \mathrm{w})$ was stirred and cooled down to $0^{\circ} \mathrm{C}$. Then, a solution of sodium nitrite $(15.00$ $\mathrm{g}, 0.217 \mathrm{~mol})$ in $45 \mathrm{ml}$ of water was added dropwise. The mixture was stirred for an additional $30 \mathrm{~min}$ at $0^{\circ} \mathrm{C}$, and the precipitate was filtered off. The resulting filtrate was then added dropwise to a solution of ammonium sulfite (59.02 g, $0.440 \mathrm{~mol}$ ) in $125 \mathrm{ml}$ of water which was previously cooled down to $0^{\circ} \mathrm{C}$, forming a yellow precipitate. The mixture was stirred for $1 \mathrm{~h}$ at $0^{\circ} \mathrm{C}$, and the precipitate was filtered off. The resulting filtrate was added to $60 \mathrm{ml}$ of hydrochloric acid (36 $\% \mathrm{w}$ ) and heated to reflux until a red solid formed and redissolved. The solution was then allowed to cool to room temperature, during which pink crystals formed, and left at $5^{\circ} \mathrm{C}$ overnight. The crystals were recovered by filtration, washed with a small amount of $1 \mathrm{M}$ hydrochloric acid and dried for $4 \mathrm{~h}$ at $50^{\circ} \mathrm{C}$ under vacuum to give the final product $(25.66 \mathrm{~g}, 57 \%)$. m.p. was not measured: degradation occurs at $230^{\circ} \mathrm{C} .{ }^{1} \mathrm{H}$ NMR (400 MHz, DMSO): $\delta$ (ppm) 10.41 (s, 3H), 8.50 (s, 1H), 7.47 - 
$7.40(\mathrm{~m}, 2 \mathrm{H}), 6.99-6.93(\mathrm{~m}, 2 \mathrm{H}) .{ }^{13} \mathrm{C} \mathrm{NMR}(101 \mathrm{MHz}$, DMSO): $\delta(\mathrm{ppm})$ 145.04, 131.54, 116.51, 112.70. FT-IR (ATR): $v=3203,2937,2669,1581,1487,1406,1164,1078$, $1012,867,809,757,612 \mathrm{~cm}^{-1}$.

5-Bromo-2,3,3-trimethyl-3H-indolenine (2)

p-Bromophenylhydrazine hydrochloride 1 (25.55 g, $0.114 \mathrm{~mol})$, 3-methyl-2-butanone (32 ml, $0.300 \mathrm{~mol})$ and $160 \mathrm{ml}$ of acetic acid were heated up to reflux for $3 \mathrm{~h}$. The solution was then allowed to cool down to room temperature, during which a red precipitate formed. The volatile components were removed under vacuum. $50 \mathrm{ml}$ of water was added, and the mixture was extracted 3 times with $40 \mathrm{ml}$ of diethyl ether, followed by 4 washing with $30 \mathrm{ml}$ of water. The combined organic phases were dried $\left(\mathrm{MgSO}_{4}\right)$, filtered on celite and the solvent was removed under vacuum. The resulting red oil was purified by distillation under vacuum (b.p. $140^{\circ} \mathrm{C}$ ) to give the final product (2) as a pale yellow oil which turned into light pink-orange crystals after few hours $(21.78 \mathrm{~g}, 80 \%)$.

m.p. $<50^{\circ} \mathrm{C} .{ }^{1} \mathrm{H}$ NMR $\left(400 \mathrm{MHz}, \mathrm{CDCl}_{3}\right): \delta(\mathrm{ppm}) 7.44(\mathrm{~m}$, $2 \mathrm{H}), 7.42-7.41(\mathrm{~m}, 1 \mathrm{H}), 2.32(\mathrm{~s}, 3 \mathrm{H}), 1.32(\mathrm{~s}, 6 \mathrm{H}) .{ }^{13} \mathrm{C} \mathrm{NMR}$ $\left(101 \mathrm{MHz}, \mathrm{CDCl}_{3}\right): \delta$ (ppm) $188.55,152.75,147.91,130.75$, 124.95, 121.36, 118.96, 54.23, 23.03, 15.51. FT-IR (ATR): $v=$ 3050, 2960, 2924, 2867, 1571, 1447, 1246, 1199, 1051, 911, $819,675,537 \mathrm{~cm}^{-1}$.

5-Bromo-1-hexadecyl-2,3,3-trimethyl-3H-indolium iodide (3)

2 (10.00 g, $42.0 \mathrm{mmol}$ ), 1-iodohexadecane (19.80 g, 56.2 $\mathrm{mmol}$ ) and $30 \mathrm{ml}$ of nitromethane were bubbled under argon and then heated to reflux overnight. The solvent was removed under vacuum, and $70 \mathrm{ml}$ of diethyl ether was added. The solution was cooled down at $5^{\circ} \mathrm{C}$ for $6 \mathrm{~h}$, and a precipitate formed, which was recovered by filtration and rinsed with cold diethyl ether. The resulting solid (3) was dried at $40^{\circ} \mathrm{C}$ under vacuum to yield a light brown powder (15.15 g, $61 \%)$.

m.p. $154^{\circ} \mathrm{C} .{ }^{1} \mathrm{H}$ NMR $\left(400 \mathrm{MHz}, \mathrm{CDCl}_{3}\right): \delta$ (ppm) $7.73-7.67$ $(\mathrm{m}, 2 \mathrm{H}), 7.61(\mathrm{~d}, \mathrm{~J}=8.5 \mathrm{~Hz}, 1 \mathrm{H}), 4.66$ (t, J = 7.4 Hz, 2H), 3.09 (s, 3H), $1.95-1.85(\mathrm{~m}, 2 \mathrm{H}), 1.67(\mathrm{~s}, 6 \mathrm{H}), 1.48-1.17(\mathrm{~m}, 26 \mathrm{H})$, $0.87(\mathrm{t}, \mathrm{J}=6.9 \mathrm{~Hz}, 3 \mathrm{H}) .{ }^{13} \mathrm{C} \mathrm{NMR}\left(101 \mathrm{MHz}, \mathrm{CDCl}_{3}\right): \delta(\mathrm{ppm})$ $195.87,143.65,140.22,132.88,126.93,124.67,117.19,54.91$, $50.70,32.02,29.79,29.78,29.75,29.72,29.66,29.57,29.45$, 29.42, 29.24, 28.00, 26.93, 23.29, 17.37, 14.22. FT-IR (ATR): $v=2917,2849,1583,1467,1413,1254,1129,986,935,872$, $822,796,719 \mathrm{~cm}^{-1}$.

2,3,3-trimethyl-5-(4,4,5,5-tetramethyl-1,3,2-dioxaborolan-2yl)-3H-indolenine (4)

2 (3.01 g, $12.6 \mathrm{mmol})$, bis(pinacolato)diboron (4.80 g, 18.9 $\mathrm{mmol})$, anhydrous potassium acetate $(3.72 \mathrm{~g}, 37.9 \mathrm{mmol})$ and dichloro[1,1'-bis(diphenylphosphino)-ferrocen]-palladium(II)

$(0.37 \mathrm{~g}, 0.5 \mathrm{mmol})$ were introduced in a $500 \mathrm{ml}$ flask, and degassed three times with vacuum/argon cycles. Dry dioxane $(200 \mathrm{ml})$ was then added through a dropping funnel under argon, and the mixture was stirred and heated up to $90^{\circ} \mathrm{C}$ for $5 \mathrm{~h}$, during which a dark brown coloration in solution appeared. The solid residues in the reaction mixture were filtered off over a thin silica layer and the solvent was removed under vacuum. Flash chromatography with $\mathrm{CH}_{2} \mathrm{Cl}_{2}: \mathrm{Et}_{3} \mathrm{~N}$ as eluent (99:1) yielded the pure product (4) as a pink powder (3.21 g, $89 \%)$. m.p. $121{ }^{\circ} \mathrm{C} .{ }^{1} \mathrm{H}$ NMR $\left(400 \mathrm{MHz}, \mathrm{CDCl}_{3}\right): \delta(\mathrm{ppm}) 7.79(\mathrm{dd}, J$ $=7.7,1.1 \mathrm{~Hz}, 1 \mathrm{H}), 7.72(\mathrm{~s}, 1 \mathrm{H}), 7.52(\mathrm{~d}, J=7.7 \mathrm{~Hz}, 1 \mathrm{H}), 2.29$ $(\mathrm{s}, 3 \mathrm{H}), 1.35(\mathrm{~s}, 12 \mathrm{H}), 1.30(\mathrm{~s}, 6 \mathrm{H}) \cdot{ }^{13} \mathrm{C}$ NMR $(101 \mathrm{MHz}$, $\left.\mathrm{CDCl}_{3}\right): \delta(\mathrm{ppm})$ 189.83, 156.46, 145.01, 135.01, 127.51, $119.46,83.85,24.98,23.13,15.63$. FT-IR (ATR): $v=2955$, $1615,1575,1479,1425,1371,1351,1329,1300,1252,1208$, 1148, 1093, 1061, 961, 911, 854, 833, 776, 706, 685, $582 \mathrm{~cm}^{-1}$. 2,5-Bis[(5-bromo-1-hexadecyl-3,3-dimethyl-2,3-dihydroindole2-ylidene)methyl]cyclobutenediylium-1,3-diolate (5)

3 (11.60 g, $19.6 \mathrm{mmol}), 3$,4-dihydroxy-3-cyclobutene-1,2-dione (squaric acid, $1.03 \mathrm{~g}, 9 \mathrm{mmol})$, quinoline $(0.51 \mathrm{~g}, 4 \mathrm{mmol})$ and $10 \mathrm{ml}$ of a 1-butanol/toluene mixture (1:1 vol) were bubbled under argon and then heated to reflux overnight with a DeanStark trap. The solution turned green in the first minutes of the reaction, and then deep blue. Solvent was removed under vacuum. The raw product was purified by flash chromatography (petroleum ether/ethyl acetate 4:1) to yield a shiny red sticky solid. A subsequent recrystallization in diethyl ether followed by a drying under vacuum at $40^{\circ} \mathrm{C}$ overnight afforded the final product (5) as shiny green crystals $(8.44 \mathrm{~g}$, $43 \%)$.

m.p. $82{ }^{\circ} \mathrm{C} .{ }^{1} \mathrm{H}$ NMR $\left(400 \mathrm{MHz}, \mathrm{CDCl}_{3}\right): \delta(\mathrm{ppm}) 7.44(\mathrm{~d}, \mathrm{~J}=$ $1.8 \mathrm{~Hz}, 2 \mathrm{H}), 7.41(\mathrm{dd}, \mathrm{J}=8.3,1.9 \mathrm{~Hz}, 2 \mathrm{H}), 6.84(\mathrm{~d}, \mathrm{~J}=8.4 \mathrm{~Hz}$, $2 \mathrm{H}), 5.96(\mathrm{~s}, 2 \mathrm{H}), 3.94(\mathrm{~s}, 4 \mathrm{H}), 1.77(\mathrm{~s}, 12 \mathrm{H}), 1.65(\mathrm{br}, 4 \mathrm{H})$, $1.44-1.18(\mathrm{~m}, 52 \mathrm{H}), 0.87(\mathrm{t}, \mathrm{J}=6.8 \mathrm{~Hz}, 6 \mathrm{H}) .{ }^{13} \mathrm{C}$ NMR $(101$ $\left.\mathrm{MHz}, \mathrm{CDCl}_{3}\right): \delta$ (ppm) 181.98, 170.10, 144.40, 141.61, 130.91, $125.87,117.01,111.02,87.53,77.36,49.55,44.19,32.06$, $31.06,29.84,29.83,29.81,29.79,29.78(\mathrm{~s}), 29.73,29.66$, 29.60, 29.49, 27.19, 27.15, 27.09, 22.83, 14.26. FT-IR (ATR): $v=3054,3023,2914,2847,1604,1474,1446,1413,1349$, $1269,1185,1066,958,919,843,792,719,689,665,565 \mathrm{~cm}^{-1}$. HRMS $(E I+, m / z)[M]+$ calculated $(\%)$ for $\mathrm{C}_{58} \mathrm{H}_{86} \mathrm{Br}_{2} \mathrm{~N}_{2} \mathrm{O}_{2}$ : 1000.5056, found 1000.5031.

2,5-bis(2,3,3-trimethyl-3H-indol-6-yl)thiophene (6)

The previously prepared 5-bromo-2,3,3-trimethyl-3H$\left.\begin{array}{llllll}\text { indolenine } & 2 & (1.61 \quad \mathrm{~g}, & 3.1 \quad \mathrm{mmol}\end{array}\right), \quad 2,5-$ bis(tributylstannyl)thiophene $(2.02 \mathrm{~g}, \quad 6.8 \mathrm{mmol})$ and tetrakis(triphenylphosphine)palladium $(0)(67 \mathrm{mg}, 0.06 \mathrm{mmol})$ were degassed via three vacuum/argon cycles, and $5 \mathrm{~mL}$ of a degassed toluene/DMF mixture (9:1 vol.:vol.) was added under argon. The reaction mixture was refluxed under argon overnight. $10 \mathrm{ml}$ of a $10 \mathrm{wt}$. \% KF aqueous solution was added, and the mixture was stirred for $1 \mathrm{~h}$ at room temperature. The resulting white precipitate was filtered off, rinsed with toluene and the filtrate was washed three times with $20 \mathrm{ml}$ of water. The organic phase was dried with $\mathrm{MgSO}_{4}$ and the solvent was removed under vacuum to give the crude product as an orange solid. A subsequent purification by flash chromatography (toluene:EtOH 96:4) followed by drying at $40^{\circ} \mathrm{C}$ under vacuum overnight afforded the pure compound (6) as orange crystals (0.84 g, $69 \%)$.

m.p. $173^{\circ} \mathrm{C} .{ }^{1} \mathrm{H}$ NMR $\left(400 \mathrm{MHz}, \mathrm{CDCl}_{3}\right): \delta(\mathrm{ppm}) 7.59(\mathrm{dd}, \mathrm{J}=$ 8.0, $1.8 \mathrm{~Hz}, 2 \mathrm{H}), 7.55-7.51(\mathrm{~m}, 4 \mathrm{H}), 7.28(\mathrm{~s}, \mathrm{~J}=3.7 \mathrm{~Hz}, 2 \mathrm{H})$, $2.30(\mathrm{~s}, 6 \mathrm{H}), 1.35(\mathrm{~s}, 12 \mathrm{H}) .{ }^{13} \mathrm{C}$ NMR $\left(101 \mathrm{MHz} \mathrm{CDCl}_{3}\right) \delta$ (ppm) 188.56, 153.38, 146.62, 143.75, 131.69, 125.42, 123.97, $120.30,118.88,53.91,23.28,15.63$. FT-IR (ATR): $v=2962$, 
$1570,1456,1423,1381,1204,1123,891,841,828,807,728$, 635, 589, 549. HRMS (EI+, m/z) [M]+ calculated $(\%)$ for $\mathrm{C}_{26} \mathrm{H}_{26} \mathrm{~N}_{2} \mathrm{~S}: 398.1817$, found 398.1799 .

\section{2,5-bis(1-hexadecyl-2,3,3-trimethyl-3H-indol-6-ium)thiophene} diiodide (7)

6 (0.80 g, $2.0 \mathrm{mmol})$, 1-iodohexadecane (7.03 g, $20.0 \mathrm{mmol})$ and $20 \mathrm{ml}$ of nitromethane were bubbled under argon and then heated to reflux overnight. The solvent was removed under vacuum, and $50 \mathrm{ml}$ of diethyl ether was added. The solution was cooled down at $5{ }^{\circ} \mathrm{C}$ overnight, and a dark brown precipitate formed, which was recovered by filtration and rinsed with cold diethyl ether. The resulting solid was dried at $40^{\circ} \mathrm{C}$ under vacuum overnight to yield a brown powder (7) $(1.83 \mathrm{~g}, 83 \%)$.

m.p. not measured, degradation occurs at $125^{\circ} \mathrm{C} .{ }^{1} \mathrm{H}$ NMR $(400$ $\left.\mathrm{MHz}, \mathrm{CDCl}_{3}\right): \delta(\mathrm{ppm}) 7.90(\mathrm{~s}, 2 \mathrm{H}), 7.74(\mathrm{dd}, \mathrm{J}=26.0,8.4 \mathrm{~Hz}$, $4 \mathrm{H}), 7.46(\mathrm{~s}, 2 \mathrm{H}), 4.61(\mathrm{t}, \mathrm{J}=6.7 \mathrm{~Hz}, 4 \mathrm{H}), 3.01(\mathrm{~s}, 6 \mathrm{H}), 1.92(\mathrm{~d}$, $\mathrm{J}=6.8 \mathrm{~Hz}, 4 \mathrm{H}), 1.73-1.67(\mathrm{~m}, 16 \mathrm{H}), 1.50-1.13(\mathrm{~m}, 52 \mathrm{H})$, $0.85(\mathrm{t}, \mathrm{J}=6.7 \mathrm{~Hz}, 6 \mathrm{H}) \cdot{ }^{13} \mathrm{C} \mathrm{NMR}\left(101 \mathrm{MHz}, \mathrm{CDCl}_{3}\right): \delta(\mathrm{ppm})$ $194.80,142.81,142.70,140.25,135.99,127.05,126.80$, $120.57,116.51,55.02,50.42,32.01,29.79,29.78,29.75,29.70$, $29.62,29.49,29.45,29.30,28.19,27.01,23.50,22.78,16.77$, 14.22. FT-IR (ATR): $v=3443,2920,2850,1608,1586,1539$, $1465,1367,1337,1297,1219,1164,1065,930,805,772,720$, $668,538 \mathrm{~cm}^{-1}$. HRMS (EI+, m/z) [M-2H]+ calculated (\%) for $\mathrm{C}_{58} \mathrm{H}_{90} \mathrm{~N}_{2} \mathrm{~S}: 848.6981$, found 846.6808 .

\section{4,7-bis(2,3,3-trimethyl-3H-indol-5-yl)benzothiadiazole (8)}

4,7-dibromobenzothiadiazole (301 mg, $1 \mathrm{mmol}), 4$ (729 mg, 2.6 mmol), tetrakis(triphenylphosphine)palladium(0) (25 mg, 0.02 mmol) and one drop of Aliquat 336 were degassed via three vacuum/argon cycles, and $10 \mathrm{~mL}$ of degassed toluene was added under argon. Then, $10 \mathrm{~mL}$ of a degassed $2 \mathrm{M}$ potassium carbonate aqueous solution was added. The reaction mixture was refluxed under argon overnight. The reaction was stopped by pouring it in $100 \mathrm{ml}$ of water, and the mixture was extracted three times with toluene. The combined organic phases were washed two times with $50 \mathrm{ml}$ of water. The organic phase was dried with $\mathrm{MgSO}_{4}$ and the solvent was removed under vacuum. A subsequent purification by flash chromatography $\left(\mathrm{CH}_{2} \mathrm{Cl}_{2}\right.$ :ethyl acetate $\left.85: 15\right)$ followed by drying at $40^{\circ} \mathrm{C}$ under vacuum overnight afforded the pure compound (8) as a yelloworange powder (384 $\mathrm{mg}, 83 \%)$.

m.p. $218^{\circ} \mathrm{C} .{ }^{1} \mathrm{H}$ NMR $\left(400 \mathrm{MHz}, \mathrm{CDCl}_{3}\right): \delta(\mathrm{ppm}) 7.93(\mathrm{dd}, \mathrm{J}=$ 8.0, $1.8 \mathrm{~Hz}, 2 \mathrm{H}), 7.91(\mathrm{~d}, \mathrm{~J}=1.2 \mathrm{~Hz}, 2 \mathrm{H}), 7.80$ (s, 2H), 7.70 (d, $\mathrm{J}=8.0 \mathrm{~Hz}, 2 \mathrm{H}), 2.35(\mathrm{~s}, 3 \mathrm{H}), 1.41(\mathrm{~s}, 7 \mathrm{H}) .{ }^{13} \mathrm{C} \mathrm{NMR}(101$ $\mathrm{MHz}, \mathrm{CDCl}_{3}$ ): $\delta$ (ppm) 189.24, 154.34, 153.93, 146.13, 134.64, $133.41,129.14,128.17,122.59,120.03,54.03,23.33,15.71$. FT-IR (ATR): $v=2954,2924,1574,1458,1428,1350,1250$, 1207, 1123, 964, 883, 843, 831,811, 600, $543 \mathrm{~cm}^{-1}$. HRMS $(\mathrm{EI}+, \mathrm{m} / \mathrm{z})[\mathrm{M}]+$ calculated for $\mathrm{C}_{28} \mathrm{H}_{26} \mathrm{~N}_{4} \mathrm{~S}$ : 450.1878 , found 450.1882 .

4,7-bis(1-hexadecyl-2,3,3-trimethyl-3H-indol-5-

ium)benzothiadiazole diiodide (9)

8 (296 mg, $0.7 \mathrm{mmol})$, 1-iodohexadecane (2.45 g, $7.0 \mathrm{mmol})$ and $8 \mathrm{ml}$ of nitromethane were bubbled under argon and then heated to reflux overnight. The solvent was removed under vacuum, and $30 \mathrm{ml}$ of diethyl ether was added. The solution was cooled down at $5^{\circ} \mathrm{C}$ overnight, and a dark brown precipitate formed, which was recovered by filtration and rinsed with cold diethyl ether. The resulting solid was dried at $40^{\circ} \mathrm{C}$ under vacuum overnight to yield a dark brown powder (9) (592 mg, $78 \%$ ).

m.p. not measured, degradation occurs at $156^{\circ} \mathrm{C} .{ }^{1} \mathrm{H}$ NMR $(400$ $\left.\mathrm{MHz}, \mathrm{CDCl}_{3}\right): \delta(\mathrm{ppm}) 8.26(\mathrm{~d}, \mathrm{~J}=1.1 \mathrm{~Hz}, 2 \mathrm{H}), 8.18(\mathrm{dd}, \mathrm{J}=$ $8.5,1.4 \mathrm{~Hz}, 2 \mathrm{H}), 8.00(\mathrm{~s}, 2 \mathrm{H}), 7.84(\mathrm{~d}, \mathrm{~J}=8.5 \mathrm{~Hz}, 2 \mathrm{H}), 4.71$ (t, $\mathrm{J}=7.2 \mathrm{~Hz}, 4 \mathrm{H}), 3.10(\mathrm{~s}, 6 \mathrm{H}), 2.03-1.91(\mathrm{~m}, 4 \mathrm{H}), 1.76(\mathrm{~s}$, $12 \mathrm{H}), 1.70-1.59(\mathrm{~m}, 4 \mathrm{H}), 1.54-1.43(\mathrm{~m}, 4 \mathrm{H}), 1.38(\mathrm{~s}, 4 \mathrm{H})$, $1.26(\mathrm{~d}, \mathrm{~J}=16.8 \mathrm{~Hz}, 40 \mathrm{H}), 0.86(\mathrm{t}, \mathrm{J}=6.9 \mathrm{~Hz}, 6 \mathrm{H}) .{ }^{13} \mathrm{C} \mathrm{NMR}$ $\left(101 \mathrm{MHz}, \mathrm{CDCl}_{3}\right): \delta$ (ppm) 195.95, 153.55, 142.23, 140.92, $139.13,131.80,130.68,129.65,124.56,115.64,55.11,50.54$, $31.98,29.75,29.74,29.71,29.66,29.58,29.45,29.41,29.27$, $28.15,26.99,23.46,22.74,17.15,14.19$. FT-IR (ATR): $v=$ $3430,2919,2850,1611,1464,1335,893,826,720 \mathrm{~cm}^{-1}$. HRMS $(\mathrm{EI}+, \mathrm{m} / \mathrm{z}) \quad[\mathrm{M}-2 \mathrm{H}]+$ calculated for $\mathrm{C}_{60} \mathrm{H}_{90} \mathrm{~N}_{4} \mathrm{~S}$ : 900.6886, found 898.6900.

Poly(bis(hexadecyl)squaraine-alt-thiophene) via Stille polycondensation (PSQT-a)

5 (502 $\mathrm{mg}, 0.5 \mathrm{mmol}), 2,5$-bis(tributylstannyl)thiophene (331 $\mathrm{mg}, 0.5 \mathrm{mmol})$ and tetrakis(triphenylphosphine)palladium $(0)$ $(15 \mathrm{mg}, 0.013 \mathrm{mmol})$ were dissolved in $10 \mathrm{~mL}$ of a degassed toluene/DMF mixture (9:1 vol.:vol.) in a $50 \mathrm{~mL}$ round-bottom flask, followed by several cycles vacuum/argon. The reaction was refluxed under argon for $48 \mathrm{~h}$. A dark green coloration of the solution could be observed. The reaction mixture was then poured into $100 \mathrm{~mL}$ of methanol. The dark purple precipitate was collected by filtration, washed with methanol, followed by purification on Soxhlet apparatus (methanol, acetone, THF and $\mathrm{CHCl}_{3}$ ). The THF and $\mathrm{CHCl}_{3}$ fractions were separately precipitated in methanol. The resulting dark purple solids were recovered by filtration and dried under vacuum at $40^{\circ} \mathrm{C}$ overnight (fraction in THF $\mathrm{m}=119 \mathrm{mg}$, fraction in $\mathrm{CHCl}_{3}$ $\mathrm{m}=293 \mathrm{mg}$, total yield : $89 \%)$.

${ }^{1} \mathrm{H}$ NMR (400 MHz, $\left.\mathrm{CDCl}_{3}\right): \delta(\mathrm{ppm}) 7.65-7.52$ (m, 4H), 7.30 $(\mathrm{s}, 2 \mathrm{H}), 6.99(\mathrm{~d}, \mathrm{~J}=5.5 \mathrm{~Hz}, 2 \mathrm{H}), 6.01(\mathrm{~s}, 2 \mathrm{H}), 4.00(\mathrm{~s}, 4 \mathrm{H}), 1.86$ $(\mathrm{s}, 12 \mathrm{H}), 1.79-1.67(\mathrm{~m}, 2 \mathrm{H}), 1.52-1.16(\mathrm{~m}, 52 \mathrm{H}), 0.87(\mathrm{t}, \mathrm{J}=$ $6.7 \mathrm{~Hz}, 6 \mathrm{H}) .{ }^{13} \mathrm{C} \mathrm{NMR}\left(101 \mathrm{MHz}, \mathrm{CDCl}_{3}\right): \delta(\mathrm{ppm})$ 169.55, $143.15,142.14,130.37,129.08,125.70,125.59,125.53$, $123.98,119.65,109.87,87.37,49.44,44.04,32.06,29.84$, $29.83,29.80,29.75,29.69,29.64,29.53,29.50,27.33,27.25$, 22.83, 14.26. FT-IR (ATR): $v=2919,2849,1599,1450,1351$, 1267, 1165, 1066, 963, 918, 788, 689, $567 \mathrm{~cm}^{-1}$.

Poly(bis(hexadecyl)squaraine-alt-benzothiadiazole) via Suzuki polycondensation (PSQBT-a)

5 (502 mg, $0.5 \mathrm{mmol}$ ), 2,1,3-Benzothiadiazole-4,7-bis(boronic acid pinacol ester) (194 $\mathrm{mg}, \quad 0.5 \quad \mathrm{mmol})$ and tetrakis(triphenylphosphine)palladium $(0)(15 \mathrm{mg}, 0.013 \mathrm{mmol})$ were dissolved in $10 \mathrm{~mL}$ of a degassed THF/toluene mixture (1:1 vol.:vol.) in a $50 \mathrm{~mL}$ round-bottom flask, followed by several cycles vacuum/argon. Then, $10 \mathrm{~mL}$ of a degassed $2 \mathrm{M}$ potassium carbonate aqueous solution was added. The reaction was refluxed under argon for $48 \mathrm{~h}$. A dark green coloration of the solution could be observed. The reaction mixture was then 
poured into $200 \mathrm{~mL}$ of methanol. The dark purple precipitate was collected by filtration, washed with water and methanol, followed by purification on Soxhlet apparatus (methanol, acetone, THF and $\mathrm{CHCl}_{3}$ ). The THF and $\mathrm{CHCl}_{3}$ fractions were separately precipitated in methanol. The resulting dark purple solid was recovered by filtration and dried under vacuum at $40^{\circ} \mathrm{C}$ overnight (fraction in THF $\mathrm{m}=115 \mathrm{mg}$, fraction in $\mathrm{CHCl}_{3}$ $\mathrm{m}=268 \mathrm{mg}$, total yield: $78 \%$ ).

${ }^{1} \mathrm{H}$ NMR (400 MHz, $\mathrm{CDCl}_{3}$ ): $\delta$ (ppm) $8.15-7.94(\mathrm{~m}, 4 \mathrm{H}), 7.84$ (s, 2H), 7.19 (d, J = 7.3 Hz, 2H), 6.16 (s, 2H), 4.10 (s, 4H), 2.09 $-1.79(\mathrm{~m}, 16 \mathrm{H}), 1.64-1.16(\mathrm{~m}, 52 \mathrm{H}), 0.87(\mathrm{t}, \mathrm{J}=6.6 \mathrm{~Hz}, 6 \mathrm{H})$. ${ }^{13} \mathrm{C}$ NMR (101 MHz, $\left.\mathrm{CDCl}_{3}\right): \delta(\mathrm{ppm}) 154.36,143.01,142.85$, $133.51,132.88,129.62,127.92,123.41,111.88,109.94,87.86$, $49.61,32.06,29.84,29.80,29.77,29.72,29.66,29.57,29.49$, 27.44, 27.27, 22.82, 14.26. FT-IR (ATR): $v=2918,2849,1599$, 1450, 1341, 1267, 1166, 1041, 965, 917, 789, 691, $565 \mathrm{~cm}^{-1}$.

Poly(bis(hexadecyl)squaraine-alt-thiophene) via metal-free polycondensation (PSQT-b)

7 (776 $\mathrm{mg}, 0.7 \mathrm{mmol})$, squaric acid (80 $\mathrm{mg}, 0,7 \mathrm{mmol})$ and quinoline $(18 \mathrm{mg}, 0,014 \mathrm{mmol})$ were added in a $25 \mathrm{ml}$ roundbottom flask which was then connected to a Dean-Stark apparatus. The reaction medium was degassed three times with vacuum/argon cycles, and $10 \mathrm{ml}$ of a toluene/1-butanol mixture (1:1 vol, bubbled under argon) was added. The mixture was then stirred and heated up to reflux for 3 days. Solubilization of the monomers was achieved only after stirring at reflux. A dark green color of the solution could be observed after few hours of reaction. The reaction mixture was then poured into cold $\mathrm{MeOH} / \mathrm{H}_{2} \mathrm{O}$ (9:1 vol.:vol.) and the resulting precipitate was filtered and purified on Soxhlet apparatus with $\mathrm{MeOH}$ (12h), acetone (24h), THF (72h) and $\mathrm{CHCl}_{3}$ (24h). A small insoluble fraction remained in the thimble and could not be recovered. The THF and $\mathrm{CHCl}_{3}$ fractions were separately concentrated under vacuum, solubilized in a minimum amount of $\mathrm{CHCl}_{3}$ and finally precipitated in $\mathrm{MeOH} / \mathrm{H}_{2} \mathrm{O}$ (9:1 vol.:vol.). The resulting dark purple solid was dried at $40^{\circ} \mathrm{C}$ under vacuum overnight (fraction in THF $\mathrm{m}=254 \mathrm{mg}$, fraction in $\mathrm{CHCl}_{3} \mathrm{~m}=167 \mathrm{mg}$, total yield: $65 \%)$.

${ }^{1} \mathrm{H}$ NMR (400 MHz, $\left.\mathrm{CDCl}_{3}\right): \delta(\mathrm{ppm}) 7.59(\mathrm{~s}, \mathrm{~J}=28.1 \mathrm{~Hz}, 4 \mathrm{H})$, $7.30(\mathrm{~s}, 2 \mathrm{H}), 7.00(\mathrm{~s}, 2 \mathrm{H}), 6.02(\mathrm{~s}, 2 \mathrm{H}), 4.23-3.66(\mathrm{~m}, \mathrm{~J}=$ $116.5 \mathrm{~Hz}, 4 \mathrm{H}), 2.01-1.63(\mathrm{~m}, 16 \mathrm{H}), 1.55-1.05(\mathrm{~m}, 52 \mathrm{H})$, $0.87(\mathrm{t}, \mathrm{J}=5.9 \mathrm{~Hz}, 6 \mathrm{H}) .{ }^{13} \mathrm{C} \mathrm{NMR}\left(101 \mathrm{MHz}, \mathrm{CDCl}_{3}\right): \delta(\mathrm{ppm})$ $169.66,143.26,142.13,130.43,125.57,124.02,119.66$, $109.90,87.38,49.45,44.08,32.06,29.84,29.80,29.75,29.69$, 29.64, 29.53, 29.50, 27.30, 27.23, 22.82, 14.26. FT-IR (ATR): $v=2919,2849,1599,1450,1351,1267,1165,1066,963,918$, $788,689,567 \mathrm{~cm}^{-1}$.

Poly(bis(hexadecyl)squaraine-alt-benzothiadiazole) via metalfree polycondensation (PSQBT-b)

9 (461 mg, $0.4 \mathrm{mmol}$ ), squaric acid (46 mg, $0.4 \mathrm{mmol}$ ) and quinoline $(10 \mathrm{mg}, 0.08 \mathrm{mmol})$ were added in a $25 \mathrm{ml}$ roundbottom flask which was then connected to a Dean-Stark apparatus. The reaction medium was degassed three times with vacuum/argon cycles, and $10 \mathrm{ml}$ of a toluene/1-butanol mixture (1:1 vol.:vol., bubbled under argon) was added. The mixture was then stirred and heated up to reflux for 3 days. Solubilization of the monomers was achieved only after stirring at reflux. A dark green color of the solution could be observed after few hours of reaction. The reaction mixture was then poured into cold $\mathrm{MeOH} / \mathrm{H}_{2} \mathrm{O}$ (9:1 vol.:vol.) and the resulting precipitate was filtered and purified on Soxhlet apparatus with $\mathrm{MeOH}$ (12h), acetone (12h), THF (48h) and $\mathrm{CHCl}_{3}$ (24h). A small insoluble fraction remained in the thimble and could not be recovered. The THF and $\mathrm{CHCl}_{3}$ fractions were separately concentrated under vacuum, solubilized in a minimum amount of $\mathrm{CHCl}_{3}$ and finally precipitated in $\mathrm{MeOH} / \mathrm{H}_{2} \mathrm{O}$ (9:1 vol.:vol.). The resulting dark purple solid was dried at $40^{\circ} \mathrm{C}$ under vacuum overnight (fraction in THF $\mathrm{m}=53 \mathrm{mg}$, fraction in $\mathrm{CHCl}_{3} \mathrm{~m}=273$ mg, total yield: $88 \%$ ).

${ }^{1} \mathrm{H}$ NMR (400 MHz, $\left.\mathrm{CDCl}_{3}\right): \delta(\mathrm{ppm}) 8.15-7.67(\mathrm{~m}, 6 \mathrm{H}), 7.18$ (s, 2H), 6.09 (s, 2H), 4.07 (s, 4H), $2.23-1.70(\mathrm{~m}, 16 \mathrm{H}), 1.60-$ $1.03(\mathrm{~m}, 52 \mathrm{H}), 0.87(\mathrm{t}, \mathrm{J}=6.8 \mathrm{~Hz}, 6 \mathrm{H}) .{ }^{13} \mathrm{C} \mathrm{NMR}(101 \mathrm{MHz}$, $\left.\mathrm{CDCl}_{3}\right): \delta(\mathrm{ppm}) 170.16,154.27,142.56,132.75,129.43$, $127.89,123.32,110.26,109.64,49.57,32.05,29.83,29.79$, $29.76,29.71,29.66,29.56,29.49,27.33,27.26,22.81,14.25$. FT-IR (ATR): $v=2918,2849,1599,1450,1341,1267,1166$, $1041,965,917,789,691,565 \mathrm{~cm}^{-1}$.

\section{Results and discussion}

\section{Synthesis of monomers}

Various monomers based on an indolenine derivative coupled with squaraine, thiophene and benzothiadazole moieties have been synthesized. General procedures for the syntheses of all the monomers are represented in Scheme 1. 


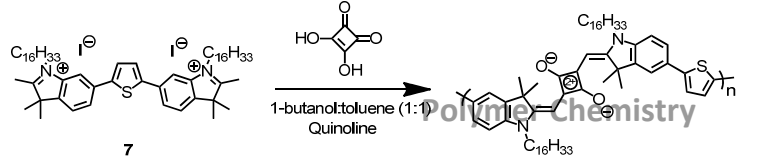

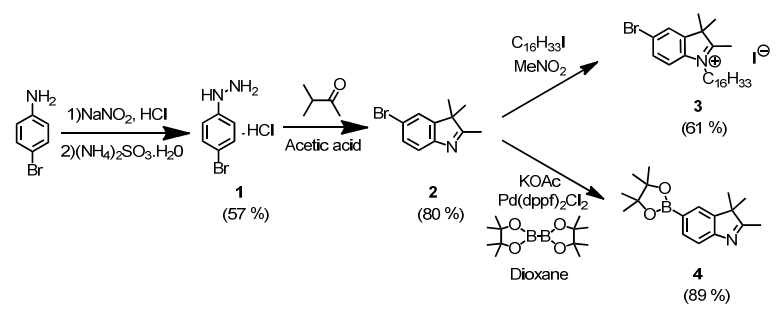
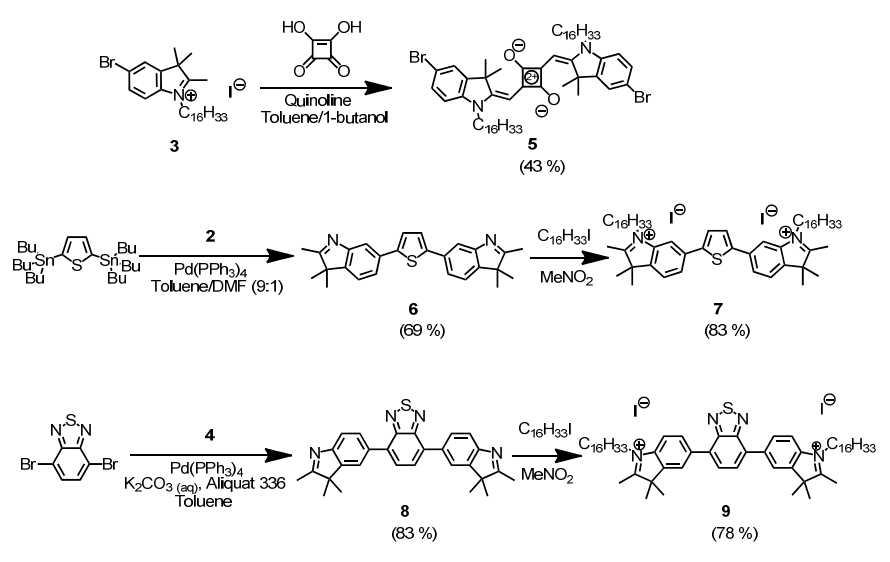

Scheme 1 Syntheses of monomers

As a starting compound for the syntheses of the different monomers, an indolenine derivative functionalized with a bromine (2) was synthesized in two steps from $p$-bromoaniline following a reported procedure. ${ }^{35}$ A subsequent quaternization of 2 with hexadecyl iodide in nitromethane afforded the indolium iodide 3 , which was then reacted with 0.45 equivalent of squaric acid in a toluene/1-butanol mixture (50/50\%vol.) to form the dibromosquaraine derivative $\mathbf{5}$, using quinoline as a base to enhance the deprotonation and reactivity of the indolium derivative 3 . The selective formation of the 1,3condensation isomer $\mathbf{5}$ as a major product was ensured by the use of mixture of toluene and 1-butanol, as already reported. ${ }^{29}$ Such monomer was already successfully used in the synthesis of polysquaraine via Yamamoto coupling. ${ }^{24}$ Bromines would provide a versatile reactivity towards usual coupling reactions employed in the synthesis of $\pi$-conjugated polymers such as Suzuki or Stille cross-coupling.

Additionally, monomers presenting a reactivity towards squaric acid were designed and synthesized to produce similar materials through metal-free polycondensation reaction. The commercially available 2,5-bis(tributylstannyl)thiophene was reacted with 2.2 equivalents of the previously synthesized bromoindole 2 by Stille cross-coupling in a mixture of toluene and dimethylformamide (DMF), using tetrakis(triphenylphosphine)palladium $(0)$ as a catalyst. The obtained compound $\mathbf{6}$ was then alkylated by quaternization of the nitrogen atoms with hexadecyl iodide in nitromethane to produce the diindolium iodide salt 7 . Finally, commercially available 4,7-dibromobenzothiadiazole was reacted with a previously synthesized boronic ester derivative of indolenine (4) in Suzuki conditions to afford compound 8. An alkylation reaction was then performed in similar conditions than the previously employed one to form compound $\mathbf{9}$.

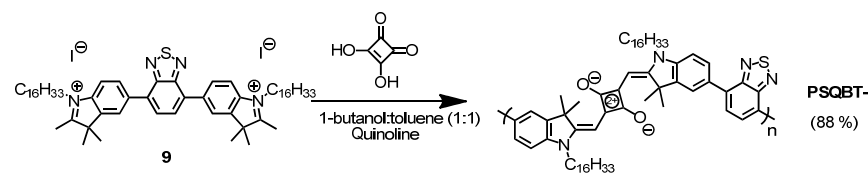

Scheme 3 Syntheses of Poly(squaraine-alt-thiophene) and poly(squaraine-alt-benzothiadiazole) via metal-free polycondensations.

\section{Synthesis of polymers}

In order to investigate the influence of the electronwithdrawing/donating properties of heteroaromatic subunits in a $\pi$-conjugated backbone containing squaraine moieties, two different alternated $\pi$-conjugated polymers were synthesized from the dibromosquaraine 5 (Scheme 2). First, poly(squarainealt-thiophene) (PSQT-a) was prepared via a Stille polycondensation between the monomer $\mathbf{5}$ and commercially available 2,5-bis(tributylstannyl)thiophene 0in a mixture of 90 $\%$ (vol.) of degassed toluene and $10 \%$ (vol.) of degassed DMF, using tetrakis(triphenylphosphine)palladium(0) as a catalyst. It was reported that highly polar solvents such as DMF acted as coordination ligand on the catalytic system, thus improving the reaction yields and molecular weights. ${ }^{36}$ Its mixing in small proportions with toluene provides a better solubility of the reactants and formed products. Poly(squaraine-altbenzothiadiazole) (PSQBT-a) was obtained by a Suzuki polycondensation of $\mathbf{5}$ with commercially available 4,7bis(4,4,5,5-tetramethyl-1,3,2-dioxaborolan-2-
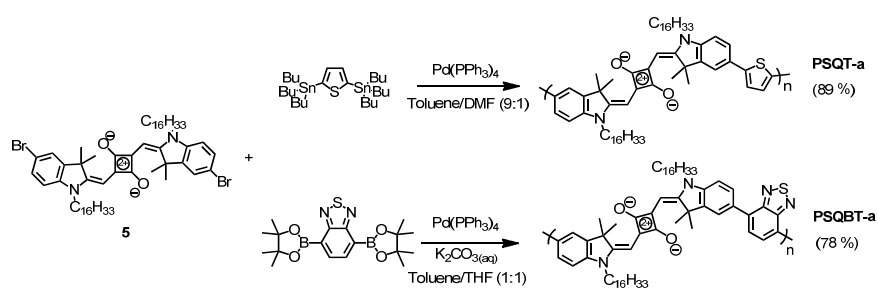

Scheme 2 Syntheses of Poly(squaraine-alt-thiophene) and poly(squaraine-alt-benzothiadiazole) via Suzuki and Stille cross-coupling.

yl)benzo[c] $[1,2,5]$ thiadiazole in a mixture of toluene and tetrahydrofuran $(50 / 50 \%$ vol.), using the same catalyst and potassium carbonate in the aqueous phase. For both polycondensations, the ratio of monomers was $1: 1$ to reach the highest molecular weights.

Similar materials were obtained through a polycondensation process without the use of transition metal (Scheme 3). The previously prepared indolium derivatives $\mathbf{7}$ and $\mathbf{9}$ were reacted with squaric acid in a 1:1 molar ratio, using a mixture of toluene and 1-butanol to favor the formation of 1,3-isomers of squaraine subunits. Additionally, the use of quinoline in small amounts combined with an azeotropic removal of the produced water helped to afford the desired PSQT-b and PSQBT-b respectively. 
The obtained materials were purified by Soxhlet extraction using methanol, acetone, tetrahydrofuran (THF) and chloroform $\left(\mathrm{CHCl}_{3}\right)$ as described in experimental part. Molecular weights of the THF and $\mathrm{CHCl}_{3}$ fractions were measured by size exclusion chromatography (SEC) using $\mathrm{CHCl}_{3}$ as an eluent (Table 1). As expected, the THF fractions corresponded to lower molecular weights than the $\mathrm{CHCl}_{3}$ ones. However, it appeared that the polycondensations of the diindolium derivatives $\mathbf{7}$ and $\mathbf{9}$ with squaric acid lead to polymers with longer chain lengths than their analogues obtained by Stille or Suzuki polycondensations, which provides an additional advantage of this type of reaction for the synthesis of $\pi$ conjugated polymers. The increase of the chain length was particularly remarkable in the case of benzothiadiazole-based monomer 9. This was attributed to an activation of the deprotonation step on the indolium moieties due to a stabilization of the generated negative charges by the electronwithdrawing benzothiadiazole core. Moreover, the selective formation of 1,3-connections in the case of PSQT-b and PSQBT-b was confirmed by the absence or near absence of peaks corresponding to carbonyl functions in the 1680-1840 $\mathrm{cm}^{-1}$ region in the infra-red absorption spectra. ${ }^{37}$

Table 1 Molecular weights ${ }^{a}$ and thermal properties ${ }^{b}$ of the synthesized materials

\begin{tabular}{|c|c|c|c|c|c|c|c|c|c|}
\hline \multirow[b]{2}{*}{ Polymer } & \multirow[b]{2}{*}{ Soxhlet fraction } & \multicolumn{4}{|c|}{ Stille/Suzuki polycond. } & \multicolumn{4}{|c|}{ Metal-free polycond. } \\
\hline & & $\begin{array}{c}\overline{\mathbb{R}_{H}} \\
\text { g.mol }{ }^{-1}\end{array}$ & Đ & $\begin{array}{l}\mathrm{T}_{5 \%} \% \\
{ }^{\circ} \mathrm{C}\end{array}$ & $\begin{array}{l}\mathrm{T}_{\mathrm{g}} \\
/{ }^{\circ} \mathrm{C}\end{array}$ & $\begin{array}{c}\overline{M_{m}} \\
/ \mathrm{g} \cdot \mathrm{mol}^{-1}\end{array}$ & Đ & $\begin{array}{l}\mathrm{T}_{5 \%} \% \\
{ }^{\circ} \mathrm{C}\end{array}$ & $\begin{array}{l}\mathrm{T} g \\
{ }^{\circ}{ }^{\circ} \mathrm{C}\end{array}$ \\
\hline \multirow{2}{*}{ PSQT } & THF & 9300 & 2.5 & - & - & 12100 & 2.2 & - & - \\
\hline & $\mathrm{CHCl}_{3}$ & 19900 & 2.7 & 353 & 26 & 23600 & 2.0 & 352 & 20 \\
\hline \multirow{2}{*}{ PSQBT } & THF & 5800 & 1.7 & - & - & 8500 & 2.3 & - & - \\
\hline & $\mathrm{CHCl}_{3}$ & 13500 & 1.3 & 353 & 20 & 36500 & 2.2 & 346 & 20 \\
\hline
\end{tabular}

${ }^{a}$ Measured by SEC in $\mathrm{CHCl}_{3}$ at $30^{\circ} \mathrm{C}$, relative to PS standards; ${ }^{b}$ Obtained by TGA under $\mathrm{N} 2$ at $10^{\circ} \mathrm{C} / \mathrm{min}$ and DSC under $\mathrm{He}$ at $20^{\circ} \mathrm{C} / \mathrm{min}$.

Thermogravimetric analyses (TGA) and differential scanning calorimetry (DSC) performed on the highest molecular weight fractions showed good thermal stability with decomposition temperatures ranging from 346 to $353^{\circ} \mathrm{C}$, as well as glass transition temperatures ranging from 20 to $26^{\circ} \mathrm{C}$. As commonly observed in the case of alternated $\pi$-conjugated polymers, such materials are amorphous. All TGA and DSC data are available in supporting informations section.

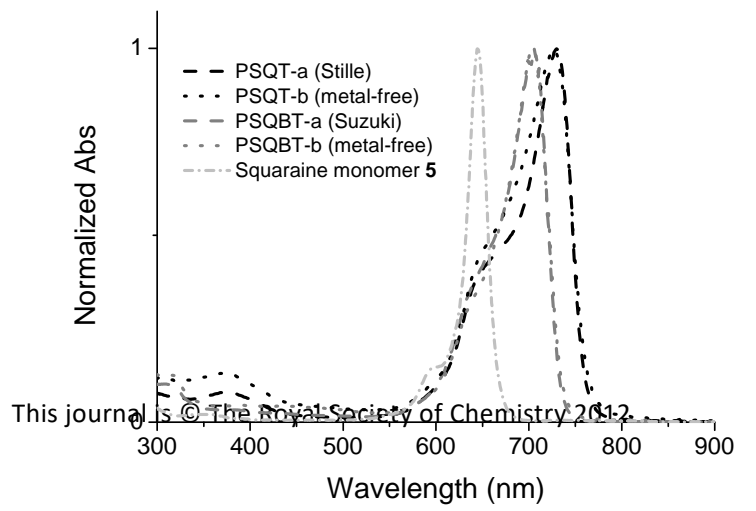

\section{Optical characterizations}

All monomers and polymers were characterized by UV-visible spectrometry. Normalized light absorption of the highest molecular weight fractions of the synthesized polymers along with monomer 5 in $\mathrm{CHCl}_{3}$ solutions are shown in Fig. 1, and the related data are reported in Table 2.

Figure 1 Normalized absorption spectra of monomer $\mathbf{5}$ and polymers in $\mathrm{CHCl}_{3}$ solutions.

$\mathrm{ICl}_{3}$ solutions $^{a}$ and solid-state ${ }^{b}$

\begin{tabular}{|c|c|c|c|c|c|c|}
\hline \multirow[b]{2}{*}{ Polymer } & \multicolumn{4}{|c|}{ Absorption } & \multicolumn{2}{|c|}{ Emission } \\
\hline & $\begin{array}{c}\lambda_{\max }{ }^{\text {sol }} \\
/ \mathrm{nm}\end{array}$ & $\begin{array}{c}\lambda_{\text {onset }^{\text {sol }}} \\
/ \mathrm{nm}\end{array}$ & $\begin{array}{c}\lambda_{\max }{ }^{\text {film }} \\
/ \mathrm{nm}\end{array}$ & $\begin{array}{c}\lambda_{\text {onset }}^{\text {film }} \\
/ \mathrm{nm}\end{array}$ & $\begin{array}{c}\lambda_{\max }{ }^{\text {sol }} \\
/ \mathrm{nm}\end{array}$ & $\begin{array}{l}\text { Stokes } \\
\text { shift sol } \\
/ \mathrm{nm}^{\mathrm{c}}\end{array}$ \\
\hline PSQT-a & 732 & 756 & 739 & 783 & 751 & 19 \\
\hline PSQT-b & 727 & 762 & 753 & 790 & 740 & 13 \\
\hline PSQBT-a & 706 & 732 & 726 & 767 & 729 & 23 \\
\hline PSQBT-b & 704 & 735 & 735 & 776 & 726 & 22 \\
\hline
\end{tabular}

${ }^{a}$ Measured on the $\mathrm{CHCl}_{3}$ fractions in $\mathrm{CHCl}_{3}$ solutions $\left(0.01 \mathrm{~g}^{-1}\right)$; ${ }^{b}$ Measured on the $\mathrm{CHCl}_{3}$ fractions in spin-coated thin films on glass substrates; ${ }^{\mathrm{c}} \lambda_{\max }$ abs. $\lambda_{\max \text { em. }}$

All the polymers showed a very pronounced red-shift when compared to the single squaraine monomer 5, confirming the extended conjugation. A similar effect was observed in the homopolysquaraine developed by Lambert and co-workers, ${ }^{24}$ indicating that the $\pi$-electrons delocalization along several squaraine dyes was mainly responsible for this red-shift. When comparing the reported bridged polysquaraines in the literature with the non-bridged polymer of Lambert and coll., a strong blue-shift of the UV-visible absorption was observed (shift of $\lambda_{\max }$ between 50 and $\left.124 \mathrm{~nm}\right){ }^{28,}{ }^{32}$ Here the absorptions were only slightly blue-shifted (shift of $\lambda_{\max }$ between 6 and $22 \mathrm{~nm}$ ). This could be attributed to a decrease in the steric hindrance generated by the bridging units, thus reducing the dihedral angle between repeating units and increasing the planarity of the backbone. The thiophene unit is expected to induce less steric hindrance than the benzothiadiazole which is linked through a phenyl ring. Hence, the absorption maxima of the corresponding polymers in solution are shifted to longer wavelength when the steric effect is reduced $\left(\lambda_{\max }\right.$ PSQBT $<$ $\left.\lambda_{\max }{ }^{\mathrm{PSQT}}\right)$. Additionally, no strong differences were observed in the absorption spectra between polymers prepared by Stille and Suzuki cross-coupling reactions and the ones obtained via metal-free polycondensation with squaric acid. While this is contradictory with the observation of high molecular weights polysquaraine in the case of metal-free polycondensation, one can argue that the maximum extent of conjugation was already reached in the shorter polymer chains obtained via metalcatalyzed couplings. 
Spin-coated films of the materials showed a bathochromic shift of their absorption (see supporting informations for details), in addition to a broadening of the peaks. The PSQs were found to have optical band-gap values around $1.6 \mathrm{eV}$ (calculated from the onset of absorption in solid state), which will be compared later with cyclic voltammetry measurements. As the absorption spectra look quite similar, it can be concluded that no (or weak) intramolecular charge transfer (ICT) involving the bridging unit takes place, whether the bridging unit is electron-donating or withdrawing.

After we have checked that we obtained similar emission spectra whatever the excitation wavelengths (i.e. $650 \mathrm{~nm}$ to 720 $\mathrm{nm}$ ), PL spectra were recorded with $\lambda_{\text {excitation a }} 650 \mathrm{~nm}$ (see Fig. 2). The small Stokes shift values, which correspond to the difference between the absorption and emission maxima, could be explained by small structural differences between the ground and excited state in the polysquaraines. It is noteworthy that PSQT-a presented the most red-shifted spectrum.

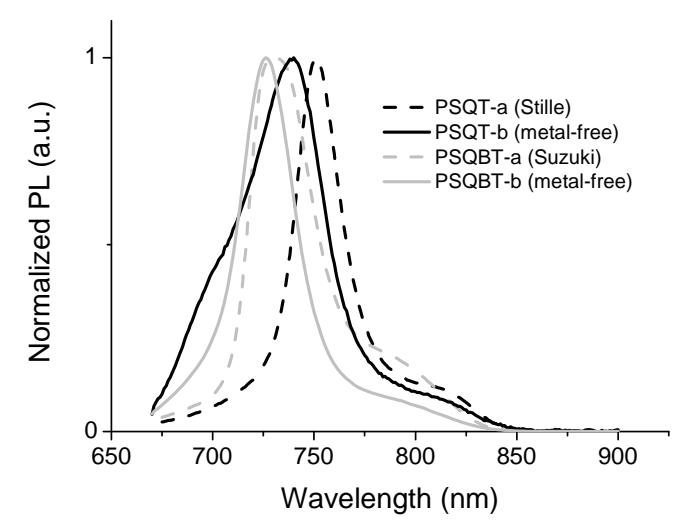

Figure 2 Normalized photoluminescence spectra of polymers in $\mathrm{CHCl}_{3}$ solutions excited at $650 \mathrm{~nm}$.

\section{Electrochemical characterizations}

Cyclic voltammetry measurements $(\mathrm{CV})$ were performed in order to determine the HOMO and LUMO levels positions along with the electrochemical band gap of the various polysquaraines. Data are gathered in Table 3 (see supporting informations for more details). CV was carried out on 0.1 g. $1^{-1}$ solutions of the materials in $\mathrm{CHCl}_{3}$, using $\mathrm{TBAPF}_{6}$ as electrolyte, silver wire as reference electrode and platinum for the working and counter-electrodes.
Table 1 Electrochemical data ${ }^{a}$ and optical band-gaps ${ }^{b}$ of the synthesized polysquaraines

\begin{tabular}{ccccc}
\hline Polymer & $\mathrm{E}_{\mathrm{HOMO}} / \mathrm{eV}$ & $\mathrm{E}_{\mathrm{LUMO}} / \mathrm{eV}$ & $\mathrm{E}_{\mathrm{g}}{ }^{\mathrm{CV}} / \mathrm{eV}$ & $\mathrm{E}_{\mathrm{g}}{ }^{\mathrm{opt}} / \mathrm{eV}$ \\
\hline PSQT-a & -5.1 & -3.6 & 1.5 & 1.6 \\
PSQT-b & -5.0 & -3.7 & 1.1 & 1.6 \\
PSQBT-a & -5.1 & -3.7 & 1.4 & 1.7 \\
PSQBT-b & -5.1 & -3.7 & 1.4 & 1.7
\end{tabular}

${ }^{a}$ Calculated from the onset of the oxidation peak $\left(E_{\text {onsetox }}\right)$ and reduction peak $\left(E_{\text {onsetred }}\right) ;{ }^{b}$ Optical band-gaps in solution calculated from $\lambda_{\text {onset }}{ }^{\text {sol }}$.

Two reversible oxidation peaks could be identified, as already reported for similar conjugated polymers,${ }^{24}$ corresponding to the formation of dicationic species. However, the reduction showed only one irreversible peak. The differences between electrochemical and optical band-gaps were explained by localized reduction and oxidation sites in the $\pi$-conjugated backbone.

A preliminary study was performed in order to investigate the photovoltaic performance of PSQBT materials. Bulk heterojunction solar cell devices were fabricated and evaluated with different ratio between PSQBT and PCBM. Unfortunately, first tests were not satisfactory. The highest power conversion efficiency (PCE) was obtained when the blend was composed of 10 wt. $\%$ of PSQBT reaching $0.4 \%$ (see Supporting Information for more preliminary results).

\section{Conclusion}

In this work we successfully synthesized small or low band-gap $\pi$-conjugated polymers based on squaraines units alternated with thiophene or benzothiadiazole moieties. We first carried out typical Stille and Suzuki cross-coupling reactions conditions, then for the sake of comparison we achieved a metal-free polycondensation reaction involving squaric acid. Interestingly, the metal-free polymerization led to higher molecular weights polysquaraines than the classical cross coupling reactions (Suzuki and Stille). Moreover, it paves the way for environement friendly routes towards $\pi$-conjugated (co)polymers with higher purity, which is crucial for optoelectronic applications.

The materials demonstrated light absorption and emission in the red-near IR. It appears that the electron-donating or electronwithdrawing character of the bridging unit (thiophene or benzothiadiazole) between squaraine moieties is of small influence on the optical properties. Instead, the steric hindrance seems to be the driving parameter in this case. Electrochemical measurements confirmed narrow band-gap materials, as well as suitably located HOMO and LUMO levels for further photovoltaic applications. Preliminary studies on polymers:PCBM blends (fluorescence quenching and surface morphology of the blends) show encouraging results for future utilization of these materials as donor in the active layer of bulk heterojunction solar cells. Further characterizations, such as measurements of the charge carrier mobility, are in progress.

More importantly, this work opens the way to easy processable, metal-free and more environmental-friendly $\pi$-conjugated 
polymers, which remain the issues and challenges in the coming next years in the field of energy transition.

\section{Acknowledgements}

The authors acknowledge financial support from the Centre National de la Recherche Scientifique, University of Bordeaux, Institut Polytechnique de Bordeaux and the Aquitaine region. They also thank Ms A.-L. Wirotius for her aid with the NMR analyses as well as Dr M. Berry for the constructive discussions about squaraine dyes.

\section{Notes and references}

${ }^{a}$ Centre National de la Recherche Scientifique (CNRS), Laboratoire de Chimie des Polymères Organiques (LCPO), UMR 5629, 16 Avenue PeyBerland, 33607 Pessac Cedex, France.

${ }^{b}$ University of Bordeaux, Laboratoire de Chimie des Polymères Organiques (LCPO), UMR 5629, 16 Avenue Pey-Berland, 33607 Pessac Cedex, France.

${ }^{c}$ Institut Polytechnique de Bordeaux (IPB), Laboratoire de Chimie des Polymères Organiques (LCPO), UMR 5629, 16 Avenue Pey-Berland, 33607 Pessac Cedex, France.

$\dagger$ Authors for correspondence: eric.cloutet@enscbp.fr and Cyril.brochon@enscbp.fr.

Electronic Supplementary Information (ESI) available: See DOI: $10.1039 / \mathrm{b} 000000 \mathrm{x} /$

1. S. Paek, H. Choi, C. Kim, N. Cho, S. So, K. Song, M. K. Nazeeruddin and J. Ko, Chemical Communications, 2011, 47, 2874-2876.

2. H. Bürckstümmer, N. M. Kronenberg, K. Meerholz and F. Würthner, Organic Letters, 2010, 12, 3666-3669.

3. J. Y. Li, C. Y. Chen, C. P. Lee, S. C. Chen, T. H. Lin, H. H. Tsai, K. C. Ho and C. G. Wu, Organic Letters, 2010, 12, 5454-5457.

4. J. Kalinowski, Optical Materials, 2008, 30, 792-799.

5. D. B. Romero, M. Schaer, M. Leclerc, D. Adès, A. Siove and L. Zuppiroli, Synthetic Metals, 1996, 80, 271-277.

6. D. Chandran and K.-S. Lee, Macromol. Res., 2013, 21, 272-283.

7. K. Sanghoon, G. K. Mor, M. Paulose, O. K. Varghese, K. Shankar and C. A. Grimes, Selected Topics in Quantum Electronics, IEEE Journal of, 2010, 16, 1573-1580.

8. A. Mishra and P. Bäuerle, Angewandte Chemie International Edition, 2012, 51, 2020-2067.

9. T. Geiger, H. Benmansour, B. Fan, R. Hany and F. Nüesch, Macromolecular Rapid Communications, 2008, 29, 651-658.

10. E. V. Agina, S. A. Ponomarenko and A. M. Muzafarov, Russ Chem Bull, 2010, 59, 1080-1098.

11. B. C. Thompson and J. M. J. Fréchet, Angewandte Chemie International Edition, 2008, 47, 58-77.

12. M. Tian, M. Furuki, I. Iwasa, Y. Sato, L. S. Pu and S. Tatsuura, The Journal of Physical Chemistry B, 2002, 106, 4370-4376.

13. C. B. Nielsen, M. Turbiez and I. McCulloch, Advanced Materials, 2013, 25, 1859-1880.
14. G. K. Dutta, A. R. Han, J. Lee, Y. Kim, J. H. Oh and C. Yang, Advanced Functional Materials, 2013, n/a-n/a.

15. B. C. Popere, A. M. Della Pelle and S. Thayumanavan, Macromolecules, 2011, 44, 4767-4776.

16. A. Treibs and K. Jacob, Angewandte Chemie International Edition in English, 1965, 4, 694-694.

17. L. Beverina, R. Ruffo, M. M. Salamone, E. Ronchi, M. Binda, D. Natali and M. Sampietro, Journal of Materials Chemistry, 2012, 22, 6704-6710.

18. S. S. Pandey, T. Inoue, N. Fujikawa, Y. Yamaguchi and S. Hayase, Thin Solid Films, 2010, 519, 1066-1071.

19. S. A. Odom, S. Webster, L. A. Padilha, D. Peceli, H. Hu, G. Nootz, S.-J. Chung, S. Ohira, J. D. Matichak, O. V. Przhonska, A. D. Kachkovski, S. Barlow, J.-L. Brédas, H. L. Anderson, D. J. Hagan, E. W. Van Stryland and S. R. Marder, Journal of the American Chemical Society, 2009, 131, 7510-7511.

20. L. Beverina and P. Salice, European Journal of Organic Chemistry, 2010, 2010, 1207-1225.

21. L. Beverina and M. Sassi, Synlett, 2014, 25, 477-490.

22. J. Eldo, E. Arunkumar and A. Ajayaghosh, Tetrahedron Letters, 2000, 41, 6241-6244.

23. A. Ajayaghosh, Chemical Society Reviews, 2003, 32, 181-191.

24. S. F. Völker, S. Uemura, M. Limpinsel, M. Mingebach, C. Deibel, V. Dyakonov and C. Lambert, Macromolecular Chemistry and Physics, 2010, 211, 1098-1108.

25. S. F. Völker, M. Renz, M. Kaupp and C. Lambert, Chemistry - A European Journal, 2011, 17, 14147-14163.

26. Q. Shi, W. Q. Chen, J. Xiang, X. M. Duan and X. Zhan, Macromolecules, 2011, 44, 3759-3765.

27. S. F. Völker and C. Lambert, Chemistry of Materials, 2012, 24, 25412553.

28. T. Maeda, T. Tsukamoto, A. Seto, S. Yagi and H. Nakazumi, Macromolecular Chemistry and Physics, 2012, 213, 25902597.

29. C. R. Chenthamarakshan, J. Eldo and A. Ajayaghosh, Macromolecules, 1999, 32, 251-257.

30. A. Ajayaghosh, Accounts of Chemical Research, 2005, 38, 449-459.

31. W. t. H. E. E. Havinga, H. Wynberg, Polymer Bulletin, 1992, 29, 119-126.

32. S. Kuster and T. Geiger, Dyes and Pigments, 2012, 95, 657-670.

33. J. Eldo and A. Ajayaghosh, Chemistry of Materials, 2001, 14, 410418.

34. A. Ajayaghosh and J. Eldo, Organic Letters, 2001, 3, 2595-2598.

35. M. V. Reddington, Bioconjugate Chemistry, 2007, 18, 2178-2190.

36. B. Carsten, F. He, H. J. Son, T. Xu and L. Yu, Chemical Reviews, 2011, 111, 1493-1528.

37. E. E. Havinga, W. ten Hoeve and H. Wynberg, Synthetic Metals, 1993, 55, 299-306. 